\title{
Role of darunavir in the management of HIV infection
}

This article was published in the following Dove Press journal:

HIVIAIDS - Research and Palliative Care

6 November 2009

Number of times this article has been viewed

\section{R Monica Lascar \\ Paul Benn \\ The Mortimer Market Centre, Camden PCT, London WCIE 6JB}

Correspondence: R Monica Lascar

The Mortimer Market Centre, Camden PCT, London WCIE 6JB, UK

Tel +442075305055

Fax +44 2075305044

Email monix@doctors.net.uk
Abstract: There is an ongoing need for potent antiretroviral therapies to deal with the increasing pool of treatment-experienced patients with multiple drug resistance. The last few years have seen the arrival of 2 new and very potent protease inhibitors - darunavir and tipranavir - alongside 2 whole new classes of anti-HIV agents - the integrase inhibitors and chemokine receptor CCR5 antagonists. This review focuses on the role of darunavir in managing HIV infection, with an emphasis on darunavir's exceptional resistance profile and related clinical effectiveness, pharmacokinetics, tolerability and toxicity data. Darunavir in combination with the pharmacokinetic booster ritonavir has proved to be very effective in the treatment of highly treatmentexperienced HIV patients with multiple drug resistance. The favorable tolerability and toxicity profile alongside the drug's high genetic barrier to the development of resistance prompted approval of darunavir for HIV-treatment naïve patients. Furthermore, the paradigm of treating HIV with a combination of anti-HIV agents is currently being challenged by ongoing darunavir monotherapy trials and these preliminary data will be discussed.

Keywords: HIV, antiretroviral therapy, darunavir

\section{Introduction}

The definition of HIV treatment success in the era of highly active antiretroviral therapy (HAART) is a moving goalpost.

Alongside well-established measures of virological success as defined by an undetectable HIV load together with an increase in CD4 T-cell count ${ }^{1,2}$ there are other drivers of treatment success, which may influence patients' and clinicians' choices of antiretroviral regimens.

Safety and tolerability of antiretroviral drugs may be as important as potency in the case of treatment-naïve patients. For the increasing pool of treatment-experienced patients, the availability of new and potent antiretroviral agents with a high genetic barrier to the development of resistance is paramount. Given the crucial role of HIV protease in the HIV replication cycle, the protease inhibitor (PI) drugs remain one of the most valuable classes of anti-HIV drugs.

This review will focus on the new PI darunavir and its role it in the treatment of HIV.

\section{Development of darunavir: pharmacology,} mode of action, pharmacokinetics, drug interactions Key steps leading to the development of TMCII4 (darunavir) The arrival of PIs in late 1996 had a major impact on reducing HIV-related morbidity and mortality. ${ }^{3}$ 
The work leading to the discovery of darunavir has been driven by several shortcomings that were evident with available antiretroviral regimes: the emergence of virological failure in up to $50 \%$ of patients treated with conventional PI regimens $s^{4}$ and the increasing prevalence of primary transmission of drug-resistant HIV variants ${ }^{5-7}$ alongside toxicity and tolerability issues associated with the relatively high therapeutic doses required. New, more potent and better-tolerated PIs were urgently needed.

The HIV-1 protease remains a major target for the development of new anti-HIV-1 drugs. This highly specific non-promiscuous enzyme cleaves the post-translational precursor polyproteins Gag and Gag-Pro-Pol in at least 9 sites, releasing 6 structural proteins (matrix, capsid, P2, nucleocapsid, P1 and P6) and 4 enzymes: protease, reverse transcriptase, RNAse and integrase. It is the essential role of protease in viral maturation ${ }^{8,9}$ that makes PIs such a crucial class of drugs for successful HIV treatment.

HIV PIs are a success of structure-based drug design. ${ }^{10}$ Our knowledge of the structure, thermodynamics and dynamics of the active site within the protease enzyme has improved greatly in the last decade, allowing for the design of 2 novel PIs - darunavir and tipranavir, ${ }^{11}$ both characterized by increased potency and higher genetic barrier to development of resistance. ${ }^{12}$

The key steps that took place in the development of darunavir, from lab bench to clinical practice, began in 1998 when a first series of PIs with superior potency were initially described. ${ }^{13}$ New insights into the molecular interactions between the PI and the viral protease enzyme were highlighted by extensive X-ray studies of protein-ligand structures of wild type and mutant HIV proteases. The backbone binding concept hypothesis ${ }^{14-16}$ has suggested that the active site backbone conformation of mutant proteases is only minimally altered. Therefore an inhibitor that maximizes interactions within the active site of HIV protease may also maintain such interactions in the presence of mutant proteases. In particular, structure-based drug design focused on increasing hydrogen-bonding interactions ${ }^{17,18}$ in the wild type enzyme, which would be difficult to overcome by mutant HIV strains, given that the enzyme backbone retains the same spatial conformation despite mutations. The way in which a drug fits within the substrate consensus volume, known as the substrate envelope, also influences drug's resilience to the development of resistance. Detailed crystal structure analyses of the binding characteristics of the HIV protease complexes has supported this hypothesis. , $^{16,19,20}$
In addition, drug design also focused on increasing the oral bioavailability of the new PIs by replacing the previous peptide ligand with non-peptidic ones, and a fused bicyclic tetrahydrofuran (bis-THF) derivate was chosen. A similar bis-THF ligand has previously been used as part of amprenavir's structure, resulting in improved bioavailability of this PI. This structural similarity between amprenavir and darunavir may be of clinical relevance in the context of development of cross-resistance between the 2 components, and these data will be discussed further.

Darunavir's exceptional resistance profile was first demonstrated in vitro, where it outperformed other available PIs by a 6- to 13 -fold difference in $\mathrm{IC}_{50}$ values. ${ }^{21,22}$ This superior potency was maintained against HIV-1 strains that were selected for saquinavir, amprenavir, indinavir, nelfinavir or ritonavir resistance, although somewhat lower activity was observed with amprenavir-resistant strains. ${ }^{21}$

Darunavir has been further developed through a unique research program, which assessed potential PI compounds against a large panel of recombinant HIV strains derived from highly PI-resistant clinical isolates. ${ }^{23}$ Following a selection process based on the drugs potency against both wild type and multi-drug resistant HIV strains alongside a favorable pharmacokinetic profile, darunavir was identified a the lead component for clinical development. ${ }^{24}$

\section{Pharmacology}

Darunavir's affinity to the HIV-1 protease is defined by a dissociation constant $\mathrm{Kd}=4.5 \times 10-12 \mathrm{M}$, which is 100 times higher than amprenavir and 1000 times higher than older PIs (indinavir, nelfinavir, ritonavir, saquinavir). ${ }^{25}$

Subsequent clinical studies ${ }^{26}$ have provided evidence on the high potency of darunavir when co-administered with low-dose ritonavir $(100 \mathrm{mg})(\mathrm{DRV} / \mathrm{r})$ both in highly PI-experienced patients as well as in treatment naïve, alongside a favorable tolerability and toxicity profile.

In vitro studies have demonstrated that darunavir is a highly potent PI, against both wild type and protease-resistant HIV strains, including a wide range of clinical isolates. This has been demonstrated with throughput cell-based screening assays, which permit assessment of wild type as well as PI-resistant HIV-1 viruses derived from clinical samples. The anti-HIV-1 in vitro activity of darunavir against wild type was demonstrated by a $50 \%$ effective concentration $\left(\mathrm{EC}_{50}\right)$ of 1 to $5 \mathrm{nM}$ and $\mathrm{EC}_{90}$ of 2.7 to $13 \mathrm{nM}$. Darunavir's potency against resistant HIV-1 was assessed against an optimization panel containing 19 recombinant clinical isolates, which were known to have resistance to other PIs 
(defined as a fold change in effective concentration - $\mathrm{EC}_{50}$ of $\geq 4 \mathrm{nM} / \mathrm{L}$ ). This demonstrated an $\mathrm{EC}_{50}<10 \mathrm{nM}$ in 18 out of 19 isolates, suggesting that darunavir would have activity against these resistant isolates. By comparison, assessing other PIs against the same panel of isolates resulted in $\mathrm{EC}_{50}$ values of $>100 \mathrm{nM}$ in the majority of specimens (nelfinavir for 19 isolates, indinavir and ritonavir for 18, saquinavir for 15 and lopinavir for 10).

Subsequently, darunavir's potency was further assessed through drug sensitivity assays on a broad range of recent clinical isolates. Among the 1501 PI-resistant samples, of which $75 \%$ had an $\mathrm{EC}_{50}<10$ nM. ${ }^{23,27}$ Cross-resistance with other PIs in vitro was not observed, with the exception of amprenavir, where darunavir was less active against HIV-1 strains selected for amprenavir resistance. ${ }^{23}$ This may be explained by the structure similarities between the 2 drugs.

An additional angle of studying a new drug's genetic barrier to resistance is through the use of biosensor-based studies, also known as surface plasmon resonance technology, which assesses the binding kinetics of the drug to the substrate. ${ }^{28}$ It is generally agreed that when resistance to PIs develops there is a decreased binding affinity, due to faster dissociation rate. In the case of darunavir a decrease of more than 1000 in binding affinity was required to see a decrease in antiviral effect, which is more than two orders of magnitude higher than observed for other PIs.

\section{Pharmacokinetics Dosage}

Darunavir has been approved for treatment of HIV infection as part of highly active antiretroviral therapy in combination with other anti-HIV agents. Given the significantly increased bioavailability of darunavir when administered together with low dose ritonavir $(100 \mathrm{mg})$, the use of unboosted darunavir is not recommended.

Darunavir is currently licensed to treat HIV-1 treatment experienced patients at a dosage of $600 \mathrm{mg}$ together with ritonavir $100 \mathrm{mg}$ twice daily (DRV/r 600/100 mg twice daily). The once daily administration of DRV/r (800/100 mg once daily) has been clinically evaluated as part of the proof of principle trial (TMC114-C207) and POWER trials in treatmentexperienced patients as well as in treatment naïve patients as part of ARTEMIS trials. Pharmacokinetic studies suggest that in treatment-naïve patients DRV/r minimum plasma concentration levels remained below the predefined EC50 of $55 \mathrm{mg} / \mathrm{mL}$, which is in keeping with clinical efficacy results (84\% HIV RNA $<50$ copies/mL at week $48 .{ }^{29}$ In the highly treatment experienced population part of POWER trials there was a trend towards a lower response rate to the once daily regimen versus the twice-daily administration (31\% vs $47 \%$, respectively). This suggests once-daily darunavir should be used with caution in highly treatment-experienced patients.

\section{Absorption, distribution, metabolism, drug interactions and special populations}

Orally administered DRV/r 600/100 mg twice daily is rapidly absorbed, reaching peak plasma concentrations within 2.5 to 4 hours. ${ }^{30}$ The terminal elimination of darunavir is relatively long at 15 hours.

Darunavir is metabolized by the hepatic cytochrome P450, in particular CYP3A. Ritonavir acts as an inhibitor of CYP3A, therefore inhibiting the hepatic first pass of darunavir, an effect which is not enhanced by further increasing the ritonavir dose to more than $100 \mathrm{mg}^{31}$

Co-administration of darunavir with other drugs metabolized via the CYP3A pathway is contra-indicated. In the setting of HIV this includes certain cholesterol lowering agents such as simvastatin, antiarrhythmics (amiodarone, quinidine) and local anesthetic lidocaine. Co-administration with other inhibitors and/or inducers of CYP3A4 is not recommended, including antituberculous drugs rifampicin and other PIs, including lopinavir.

Darunavir is mainly protein bound (95\%), ${ }^{32}$ and although no changes in total plasma darunavir concentration were reported in patients with mild or moderate liver disease, the concentration of unbound darunavir was increased in these groups. Manufacturers advise caution when using darunavir in patients with moderate liver disease and its use is contra-indicated in patients with severe liver disease. ${ }^{33}$ Co-infection with hepatitis $\mathrm{B}$ and $\mathrm{C}$ is not in itself a contraindication to using DRV/r, as reported by a substudy analysis of darunavir exposure in this group (POWER 3). ${ }^{34}$

With regards to darunavir's penetration in other compartments such as the central nervous system (CNS), there are data suggesting good penetration in the CNS at levels sufficient to inhibit viral replication for wild-type virus. ${ }^{35}$ Few data are currently available on darunavir penetration in the genital tract.

\section{Efficacy studies/clinical results}

Clinical trial data on darunavir's safety and efficacy stem from 3 main trials: POWER trials on highly treatment-experienced patients; TITAN, which included less treatment-experienced patients who were lopinavir/ritonavir (LPV/r) naïve; and ARTEMIS trials with antiretroviral treatment-naïve patients. 
Given the structure-based drug discovery and evaluation process $^{21,23,27}$ which led to the identification of darunavir as a potent agent against multi-drug resistant HIV strains in vitro, the first logical step of clinical evaluation studies to be undertaken were in highly HIV treatment experienced patients.

\section{Role of darunavir in treatment- experienced patients}

The POWER (Performance Of TMC114/ritonavir When evaluated in Treatment-Experienced patients with PI resistance) studies ${ }^{36}$ demonstrated DRV/r clinical safety and efficacy in patients with previous triple-class exposure and advanced HIV disease.

POWER 1 and 2 studies were randomized, partially blinded, dose finding studies, which compared the efficacy of $\mathrm{DRV} / \mathrm{r}$ against investigator-chosen control protease inhibitors (CPI) in addition to an optimized background therapy (OBT), in treatment-experienced patients with at least one primary PI mutation and HIV load $>1000$ copies $/ \mathrm{mL}$.

The use of enfuvirtide was permitted but tipranavir, another novel PI, was precluded as part of OBT. The clinician was only blinded to the dose of DRV/r used in the first 24 weeks (600/100 mg twice daily, 400/100 mg twice daily, 800/100 mg once daily and 400/100 mg once daily), which identified the DRV/r dose of 600/100 mg twice daily to achieve the best virological and immunological response. After this, patients receiving other doses of DRV/r were switched to $600 / 100 \mathrm{mg}$ twice daily and patients receiving control PIs continued their regimens in the open label phase of the study. Results from POWER 1 and 2 studies over time are presented in Table 1. POWER 1, 2 and 3 differed in geographical location, and inclusion of patients co-infected with hepatitis B and C (stable and without signs of chronic liver disease) was permitted in POWER 1 but not in POWER 2.

The primary end-point defined as drop in HIV viral load (VL) of at least $1 \log _{10}$ copies/mL and assessed using the time to loss of virological response algorithm (TLOVR) was achieved by $61 \%(67 / 110) \mathrm{DRV} / \mathrm{r}$ patients compared to $15 \%(18 / 120)$ in the open label CPI arm after 48 weeks of treatment ${ }^{36}$ and this difference remained statistically significant through to week $96^{37}$ and week $144^{38}(P<0.001)$. This was an impressive result considering that the patients included in the POWER trials had fairly advanced disease as defined by a median HIV load of $4.61 \log 10$ copies/mL, a low mean CD4 count of 153 cells/mL and almost all the patients had used at least 2 PIs previously, with 63\% appearing nonsensitive to commercially available PIs at that time.

The higher the number of active anti-HIV agents used, the more likely that virological suppression of less than 50 copies $/ \mathrm{mL}$ was achieved. This was also confirmed in a subsequent study, ${ }^{39}$ which showed that although the risk of viral rebound in heavily pre-treated patients was higher in the first year, this decreased significantly the longer virological suppression was maintained. The best predictor of virological response was the fold change in $\mathrm{EC}_{50}$ values compared to wild-type reference virus. ${ }^{40}$

Table I Efficacy data for darunavir/ritonavir versus control PI regimens (POWER studies) ${ }^{36}$ and lopinavir/ritonavir (TITAN ${ }^{41}$ ) in treatmentexperienced patients

\begin{tabular}{llll}
\hline POWER I & & & \\
\hline Virological response (24 wk) & DRV/r 600/100 N=60 & CPI/r N=60 & P values \\
\hline$V L<400$ & $67 \%$ & $25 \%$ & $P=0.001$ \\
$V L<50$ & $53 \%$ & $18 \%$ & $P=0.00 I$
\end{tabular}

\section{POWER 2}

Virological response $(24 \mathrm{wk})$

$\mathrm{VL}<400$

$\mathrm{VL}<50$
DRV/r 600/100 N = 39

$49 \%$

$39 \%$

DRV/r ( $\mathbf{N}=\mathbf{2 9 8})$

$77 \%$

$71 \%$
$\mathrm{CPI} / \mathrm{r} \mathrm{N}=\mathbf{4 2}$

$10 \%$

$7 \%$

$P=0.001$

$P=0.001$

\section{TITAN}

\section{Virological response (48 wk)}

$\mathrm{VL}<400$

$\mathrm{VL}<50$
LPV/r $(\mathbf{N}=\mathbf{2 9 7})$

$67 \%$

$60 \%$
$P=0.08$

$P=0.05$

Notes: Data suggest darunavir superiority as defined by HIV RNA $<50$ when compared to control Pls in the POWER trials and a trend towards superiority for LPV/r in TITAN at week 48 (clear superiority at week 96 - data not shown). The data illustrated here show virological responses to 600/I00 twice daily darunavir ritonavir dosage only. Abbreviations: $\mathrm{CPI} / \mathrm{r}$, protease inhibitor/ritonavir; DRV/r, darunavir/ritonavir; PI, protease inhibitor; VL, viral load. 
$\mathrm{DRV} / \mathrm{r}$ was further evaluated in $595 \mathrm{PI}$-experienced patients who were naïve to $\mathrm{LPV} / \mathrm{r}$ and were randomized to receive $\mathrm{DRV} / \mathrm{r}$ or $\mathrm{LPV} / \mathrm{r}$ as part of the TITAN trial (TMC114/ritonavir In Treatment-experienced pAtients Naïve to lopinavir). The primary end point was to prove non-inferiority of DRV/r (600/100 mg twice daily) vs LPV/r (400/100 mg twice daily) (VL $<400$ copies/mL at 48 weeks) and secondary endpoints included achieving a HIV VL of less than 50 copies $/ \mathrm{mL}$. Patients recruited were PI naïve in $30 \%$ of the cases, $33 \%$ had used one PI in the past and the rest had been exposed to 2 PIs and the distribution of patients was well balanced between the 2 arms. Virological response at week 48 (Table 1) showed that $77 \%$ of DRV/r patients versus $68 \%$ of LPV/r achieved a HIV VL $<400$ copies $/ \mathrm{mL}$ (estimated difference of 9\%, 95\% CI 2-16). This has demonstrated that DRV/r was non-inferior to $\mathrm{LPV} / \mathrm{r}$ and the secondary statistical analysis suggested superiority of DRV/r in this setting. Furthermore, patients recruited to the TITAN study had less advanced disease than in the POWER trials and use of enfuvirtide was not permitted. This suggests that the differences observed in the TITAN study were due to the intrinsic antiviral efficacy of DRV/r.

Further evidence on DRV/r efficacy comes from a single-center study of 109 three-class experienced patients who switched regimens to either (i) boosted darunavir, (ii) other PI or (iii) non-PI class (including raltegravir). ${ }^{42}$ Multivariate analysis suggested that patients switching to darunavir or raltegravir or both were more likely to achieve the primary outcome of HIV RNA $<50$ copies/mL at 24 weeks compared to those switching to another protease inhibitor (for darunavir $65 \%$, odds ratio $=4.24$ vs non-PI strategy). The results further confirm darunavir's efficacy as shown by company-led trials (POWER and TITAN) and provide evidence on switching strategies for highly experienced patients.

\section{Role of darunavir in treatment naïve patients}

$\mathrm{DRV} / \mathrm{r}$ at a once daily dose (800/100 mg once daily) was compared to LPV/r 800/200 mg total daily dose (15\% of total patients received LPV/r once daily) in 689 treatment naïve patients with HIV RNA > 5000 copies/mL as part of the ARTEMIS (AntiRetroviral Therapy with TMC114 ExaMined In naïve Subjects) trial. All patients received a fixed dose combination of tenofovir disoproxil fumarate and emtricitabine.

The primary outcome measure was non-inferiority of $\mathrm{DRV} / \mathrm{r}$ vs LPV/r as defined by achieving a HIV VL of less than 50 copies/mL in a TLOVR analysis at week 48 . Overall response at 48 weeks showed $84 \%$ of subjects on DRV/r vs $78 \%$ on LPV-R had a VL $<50$ copies/mL and DRV/r was non-inferior to $\mathrm{LPV} / \mathrm{r}(P<0.005) .{ }^{43}$ In the superiority analysis at week 48 the estimated difference was $5.5 \%$ in favor of DRV/r, which was not statistically significant. 36\% of patients in each arm had a HIV RNA $>100,000$ copies/mL at baseline and in this subgroup $79 \%$ of $\mathrm{DRV} / \mathrm{r}$ vs $67 \%$ on $\mathrm{LPV} / \mathrm{r}$ were undetectable at week 48 suggesting a statistical significant superiority of $\mathrm{DRV} / \mathrm{r}$ in this high HIV load subgroup.

A more recent 96 weeks analysis ${ }^{44}$ confirmed noninferiority of $\mathrm{DRV} / \mathrm{r}$ versus LPV-R in naïve patients, with a statistical difference of $8.4 \%$ and $95 \%$ confidence interval 1.9 to $14.8(P<0.001)$. In an intent-to-treat analysis $\mathrm{DRV} / \mathrm{r}$ was shown to be superior to $\mathrm{LPV} / \mathrm{r}$ at week 96 with regards to virological end point, which was achieved by $79 \%$ of patients in DRV/r versus $65 \%$ treated with $\mathrm{LPV} / \mathrm{r}$ $(P=0.012)$. However there were no significant differences in the median CD4 cell count increases from baseline within the 2 groups.

\section{Darunavir monotherapy in patients with HIV RNA $<\mathbf{5 0}$ copies/mL}

Given the proven superiority of DRV/r when compared to CPIs in treatment experienced patients in the POWER and TITAN studies as well as in comparison to $\mathrm{LPV} / \mathrm{r}$ in naïve patients (ARTEMIS), trials are currently underway to explore whether DRV/r monotherapy would be an effective way of managing HIV patients. The question being addressed is whether DRV/r monotherapy can be used as a maintenance therapy once the patient has achieved an undetectable viremia with a standard approved combination therapy. This would be a major shift in the paradigm of HIV care, where a return to an effective and tolerable anti-HIV monotherapy would potentially reduce unwanted side-effects for patients as well as costs for the care provider.

This hypothesis is currently being evaluated as part of 2 multicenter, open label randomized trials (MONET and MONOI), both recruiting patients who are stable on antiretroviral medication with an undetectable baseline HIV load, aiming to prove non-inferiority of DRV/r monotherapy versus standard HAART given as 2 nucleoside reverse transcriptase inhibitors (NRTI) together with DRV/r. ${ }^{45,46}$ Results at 48 weeks showed that DRV/r monotherapy was non-inferior to $2 \mathrm{NRTI}$ and DRV/r (MONET $\mathrm{N}=123$ patients in each arm) in a per protocol analysis, with $86.5 \%$ remaining undetectable ( $\mathrm{HIV}$ VL $<50$ copies $/ \mathrm{mL}$ ) in the 
monoterapy arm versus $87.8 \%$ in the standard treatment arm (lower limit of the $95 \% \mathrm{CI}$ at $-10.1 \%$ ). In the intent to treat analysis in $\mathrm{MONOI}^{45}(\mathrm{~N}=225$ divided in the 2 arms $)$ non-inferiority could not be demonstrated, $87.5 \%$ in the monotherapy arm versus $91 \%$ of patients in the standard had undetectable viral load at week 48 (lower limit of the $95 \%$ confidence interval at $-11 \%$ and less than the pre-established $-10 \%$ threshold). The patients included in the MONOI study had more advanced disease and the identified trend towards non-inferiority (see Table 3), suggests that longer term follow-up is required to delineate virological outcomes.

In those individuals experiencing low-level HIV virological rebound, no new darunavir-resistant mutations where detected and the reintroduction of 2 nucleoside analogues as part of HAART resulted in viral suppression below 50 copies/mL in all patients. Although these data are encouraging, longer follow-up is required to ensure that virological suppression is maintained in the longer term. There were no statistically significant differences in side effects in both groups at 48 weeks.

Furthermore, consideration to viral control in other compartments, such as CNS disease and genital tract is recommended before the use of PI monotherapy can be recommended.

\section{Safety and tolerability}

Boosted darunavir has been shown to be safe and well tolerated by treatment-experienced ${ }^{36}$ and treatment-naïve HIV patients. DRV/r was generally well tolerated by both groups of patients. Combined POWER analysis and TITAN data show that the main side effects reported in treatment experienced patients after 48 weeks of treatment were similar to previous PIs and included diarrhea, nausea, headache, naso-pharyngitis and upper respiratory tract infections. Of note, the overall frequency of various side effects in the combined POWER analysis was higher in the CPI-treated patients, in spite of the lower levels of overall drug exposure in this group (where higher rates of treatment failure lead to more frequent treatment discontinuation in the CPI group). Duration of treatment exposure was similar within the 2 arms of the TITAN study (53 vs 51 weeks). No further tolerability issues became apparent on subsequent follow-up for 144 weeks in POWER trials ${ }^{47}$ or up to week 96 follow-up from TITAN. Table 2 illustrates frequency of adverse events in treatment-naïve and treatment-experienced patients.

Most common laboratory abnormalities were elevated lipids, which occurred at similar frequencies in both arms in treatment-experienced patients (incidence of trigliceride elevations was $5 \%$ or greater in both arms in POWER trials). In treatment-naïve patients in the ARTEMIS study the overall incidence of gastro-intestinal side effects was lower in the darunavir arm, a trend which continued to week 96 and was statistically significant $(P<0.0001)$. The incidence of lipid abnormalities, both cholesterol and triglyceride elevations, was also lower in DRV/r-treated patients. Although there are no head-to-head comparisons between atazanavir, which has also been shown to have less impact on lipid profiles than other PIs and darunavir, a study in healthy volunteers found

Table 2 Safety and tolerability summary data for darunavir/ritonavir in treatment-experienced and treatment-naïve patients

\begin{tabular}{|c|c|c|}
\hline \multicolumn{3}{|c|}{ A. TITAN safety and tolerability data in treatment experienced patients ${ }^{4 I}$} \\
\hline $\begin{array}{l}\text { Grade } 2-4 \text { laboratory abnormalities } \\
\text { (incidence } \geq 2 \% \text { ) }\end{array}$ & $\mathrm{DRV} / \mathbf{r}(\mathbf{N}=\mathbf{2 9 8})$ & LPV/r $(\mathbf{N}=\mathbf{2 9 7})$ \\
\hline Diarrhea & $14(4 \%)$ & $34(10 \%)^{*}$ \\
\hline Triglycerides increased & $10(3 \%)$ & $38(11 \%)$ \\
\hline Total cholesterol increased & $44(13 \%)$ & $78(23 \%)$ \\
\hline Low-density lipoprotein increased & $44(13 \%)$ & $36(11 \%)$ \\
\hline \multicolumn{3}{|c|}{ B. ARTEMIS safety and tolerability data in treatment-naïve patients ${ }^{29}$} \\
\hline Diarrhea & $23(7.7 \%)$ & $43(14.5 \%)^{*}$ \\
\hline Triglycerides increased & $57(19 \%)$ & $75(25 \%) *$ \\
\hline Total cholesterol increased & $94(32 \%)$ & $86(29 \%)$ \\
\hline Low-density lipoprotein increased & $56(19 \%)$ & $50(17 \%)$ \\
\hline
\end{tabular}

$* P<0.05 ; * P<0.01$

Abbreviations: DRV/r, darunavir/ritonavir; LPV/r, lopinavir/ritonavir. 
Table 3 Darunavir monotherapy data

\begin{tabular}{|c|c|c|c|}
\hline \multicolumn{4}{|l|}{ A. $\mathrm{MONOI}^{45}$} \\
\hline $\begin{array}{l}\text { Virological response } \\
(48 \mathrm{wk})\end{array}$ & DRV/r & DRV/r + 2NRTI & $\begin{array}{l}\text { Difference } \\
\text { (lower limit CI) }\end{array}$ \\
\hline $\mathrm{VL}<50 \mathrm{PP}(\mathrm{n}=204)$ & $94.1 \%$ & $99 \%$ & $-4.9(-9)$ \\
\hline $\mathrm{VL}<50$ ITT $(\mathrm{n}=225)$ & $87.5 \%$ & $92 \%$ & $-4.5(-1 \mathrm{I})^{*}$ \\
\hline \multicolumn{4}{|l|}{ B. MONET $^{44}$} \\
\hline $\mathrm{VL}<50 \mathrm{PP}$ & $86.2 \%(n=123)$ & $87.8 \%(n=123)$ & $-1.6(-10.1)$ \\
\hline $\mathrm{VL}<50$ ITT & $84.3 \%(n=127)$ & $85.3 \%(n=129)$ & $-1(-9.9)^{*}$ \\
\hline \multicolumn{4}{|c|}{$\begin{array}{l}*-11 \%<-10 \% \text { (delta) failure to demonstrate non-inferiority in ITT analysis; } *-9.9 \%>-12 \% \text { (delta) showing non-inferiority of darunavir monotherapy in ITT analysis. } \\
\text { Notes: Both trials have recruited patients who are stable on antiretroviral medication with an undetectable baseline HIV load and were switched to receiving either ritonavir- } \\
\text { boosted darunavir monotherapy (DRV/rarm) versus triple therapy comprising ritonavir-boosted darunavir together with a fixed nucleoside backbone two nucleoside reverse } \\
\text { transcriptase inhibitors (DRV/r }+2 \mathrm{NRTI} \text { arm). } \\
\text { A. Primary efficacy analysis results from MONOI study at week } 48 \text { (end point: HIV RNA }<50 \text { copies } / \mathrm{mL} \text { ) show non-inferiority of darunavir monotherapy in a per protocol } \\
\text { (PP) analysis but not in the ITT analysis. Of note, the trial was powered to detect a difference 'delta' of }-10 \% \text {. } \\
\text { B. Primary efficacy results from MONET at week } 48 \text {, with HIV RNA }<50 \text { copies } / \mathrm{mL} \text { using a TLOVR algorithm (where switch equals failure) are shown. Darunavir monotherapy } \\
\text { showed consistently non-inferior efficacy compared to triple therapy at week } 48 \text {. Of note, the difference 'delta' was set at }-12 \% \text { in this trial. } \\
\text { Abbreviations: Cl, confidence interval; DRV/r, darunavir/ritonavir; NRT, nucleoside reverse transcriptase inhibitors; PP, per protocol analysis; ITT, intent to treat analysis; }\end{array}$} \\
\hline
\end{tabular}
VL, viral load.

that both PIs had only minor and similar effects on lipid profile in the short term. ${ }^{48}$

None of the studies reported significant renal abnormalities with DRV/r and the incidence of rashes was greatest in the TITAN at $3 \%$. Overall good tolerability is also reflected in the low discontinuation rates due to adverse events in the darunavir arm (3\% at 48 weeks and $4 \%$ at 96 weeks in ARTEMIS trial).

\section{Resistance to darunavir}

Darunavir's potency in vitro was demonstrated against wild type and multi-drug resistant clinical isolates. ${ }^{21,23}$ There was no cross-resistance with other PIs in vitro, although due to structural similarities with amprenavir this may potentially occur. ${ }^{21}$ Furthermore, in vitro selection studies suggested that resistance to DRV is slow to develop compared to existing PIs. ${ }^{23}$

The strongest predictor of virological response to darunavir in POWER trials was the fold change in EC50 value for darunavir at baseline ${ }^{40}$ and 2 phenotypic clinical cut off $(\mathrm{CCO})$ values were defined (low $\mathrm{CCO}=10$ and upper $\mathrm{CCO}=40$ ), which may aid clinicians with access to phenotypic information.

The number and type of mutations in the protease associated with increased fold change for darunavir were further analyzed at 24 weeks and subsequently in the POWER trials and 11 protease mutations associated with a decreased response to arunavir were identified: V11I, V32I, L33F, I47V, I50V, I54L/M, G73S, L76V, I84V and L89V, with 5 major resistance associated mutations (highlighted in bold) being more commonly observed and associated with treatment failure in highly treatment-experienced patients. Resistance to darunavir was more commonly observed when at least three darunavir resistance-associated mutations (RAMs) were present in the context of a high number $(\geq 14)$ of baseline International AIDS Society resistance associated mutations IAS-USA PI RAMs. ${ }^{37,40}$

The prevalence of darunavir RAMs was analyzed in a retrospective study of 1021 clinical isolates from a reference laboratory in Madrid. The genotypes of patients who had previously failed commercially available PIs were examined for the presence of DRV resistance-associated mutations and 3 or more were found in $6.7 \%$ of case but two-thirds of patients did not have any mutations detected.

\section{Place in therapy}

Undoubtedly, darunavir has secured a key place for use in patients with multi drug resistant HIV, where it was shown to be more potent than investigator selected PI in POWER and TITAN. Furthermore, an additional single center switch study further confirmed that three-class experienced patients switched to darunavir were more likely to achieve virological suppression than their counterparts switched to other PIs or to non-PI class. ${ }^{49}$ Use of $\mathrm{DRV} / \mathrm{r}$ in treatment experienced patients is further backed up by cost effectiveness studies, ${ }^{50,51}$ which evaluated treatment costs for HIV RNA reduction ( 0.5 log reduction) and CD4 count increases ( 25 cells rise) for boosted darunavir versus other antiretrovirals in treatment 
experienced patients, using clinical data from POWER and TITAN trials. These studies showed that although costs varied widely from US\$132 for DRV/r to US\$16,464 for T-20, the use of darunavir in this highly treatment-experienced population was cost-effective despite the higher initial purchase price when compared to older PIs. This is due to the high efficacy of darunavir and secondary increase in CD4 counts, which is associated with less HIV-related morbidity and associated health care costs. ${ }^{52}$

There are increasing data supporting the use of DRV/r in naïve patients, based on the drug's favorable safety and tolerability profile, together with medium long-term efficacy data. It may be argued that the best strategy is to use highly potent anti-HIV therapy as first line in order to prevent treatment failure rather than keeping more potent agents as second line to deal with treatment failure when this occurs. The favorable toxicity and tolerability profile in treatment-naïve patients means darunavir is well placed for first-line treatment in those with treatment resistance. Studies evaluating the performance of darunavir with other classes of anti-HIV drugs and with existing agents such as efavirenz in treatment-naïve patients are required.

The accumulating data on the use of darunavir as monotherapy are encouraging and suggest that this strategy may be used as 'maintenance' therapy in patients with previous HIV RNA $<50$ copies/mL. However, further information is required on the durability of this strategy and implications for viral evolution in other compartments (CNS, genital tract).

\section{Disclosures}

The authors declare no conflicts of interest.

\section{References}

1. Gazzard B, Bernard AJ, Boffito M, et al. British HIV Association (BHIVA) guidelines for the treatment of HIV-infected adults with antiretroviral therapy. HIV Med. 2006;7(8):487-503.

2. Hammer SM, Saag MS, Schechter M, et al. Treatment for adult HIV infection: 2006 recommendations of the International AIDS SocietyUSA panel. JAMA. 2006;296(7):827-843.

3. Palella FJ, Jr., Delaney KM, Moorman AC, et al. Declining morbidity and mortality among patients with advanced human immunodeficiency virus infection. HIV Outpatient Study Investigators. $N$ Engl J Med. 1998;338(13):853-860.

4. Grabar S, Pradier C, Le Corfec E, et al. Factors associated with clinical and virological failure in patients receiving a triple therapy including a protease inhibitor. AIDS. 2000;14(2):141-149.

5. Wainberg MA, Friedland G. Public health implications of antiretroviral therapy and HIV drug resistance. JAMA. 1998;279(24):1977-1983.

6. Brenner B, Routy JP, Quan Y, et al. Persistence of multidrugresistant HIV-1 in primary infection leading to superinfection. AIDS. 2004;18(12):1653-1660.

7. Brenner BG, Roger M, Moisi DD, et al. Transmission networks of drug resistance acquired in primary/early stage HIV infection. AIDS. 2008;22(18):2509-2515.
8. Tomasselli AG, Heinrikson RL. Targeting the HIV-protease in AIDS therapy: a current clinical perspective. Biochim Biophys Acta. 2000;1477(1-2):189-214.

9. Tomasselli AG, Heinrikson RL. Specificity of retroviral proteases: an analysis of viral and nonviral protein substrates. Methods Enzymol. 1994;241:279-301.

10. Wlodawer A. Structure-based design of AIDS drugs and the development of resistance. Vox Sang. 2002;83 Suppl 1:23-26.

11. de Mendoza C, Morello J, Garcia-Gasco P, Rodriguez-Novoa S, Soriano V. Tipranavir: a new protease inhibitor for the treatment of antiretroviral-experienced HIV-infected patients. Expert Opin Pharmacother. 2007;8(6):839-50.

12. Surleraux DL, de Kock HA, Verschueren WG, et al. Design of HIV-1 protease inhibitors active on multidrug-resistant virus. J Med Chem. 2005;48(6):1965-1973.

13. Ghosh AK, Kincaid JF, Cho W, et al. Potent HIV protease inhibitors incorporating high-affinity P2-ligands and (R)-(hydroxyethylamino)s ulfonamide isostere. Bioorg Med Chem Lett. 1998;8(6):687-690.

14. Ghosh AK, Dawson ZL, Mitsuya H. Darunavir, a conceptually new HIV-1 protease inhibitor for the treatment of drug-resistant HIV. Bioorg Med Chem. 2007;15(24):7576-7580.

15. Ghosh AK, Chapsal BD, Weber IT, Mitsuya H. Design of HIV protease inhibitors targeting protein backbone: an effective strategy for combating drug resistance. Acc Chem Res. 2008;41(1):78-86.

16. Kovalevsky AY, Louis JM, Aniana A, Ghosh AK, Weber IT. Structural evidence for effectiveness of darunavir and two related antiviral inhibitors against HIV-2 protease. J Mol Biol. 2008;384(1):178-192.

17. Hong L, Zhang XC, Hartsuck JA, Tang J. Crystal structure of an in vivo HIV-1 protease mutant in complex with saquinavir: insights into the mechanisms of drug resistance. Protein Sci. 2000;9(10):1898-1904.

18. Laco GS, Schalk-Hihi C, Lubkowski J, et al. Crystal structures of the inactive D30N mutant of feline immunodeficiency virus protease complexed with a substrate and an inhibitor. Biochemistry. 1997;36(35):10696-10708.

19. Kovalevsky AY, Liu F, Leshchenko S, et al. Ultra-high resolution crystal structure of HIV-1 protease mutant reveals two binding sites for clinical inhibitor TMC114. J Mol Biol. 2006;363(1):161-173.

20. Prabu-Jeyabalan M, Nalivaika E, Schiffer CA. Substrate shape determines specificity of recognition for HIV-1 protease: analysis of crystal structures of six substrate complexes. Structure. 2002;10(3):369-381.

21. Koh Y, Nakata H, Maeda K, et al. Novel bis-tetrahydrofuranylurethanecontaining nonpeptidic protease inhibitor (PI) UIC-94017 (TMC114) with potent activity against multi-PI-resistant human immunodeficiency virus in vitro. Antimicrob Agents Chemother. 2003;47(10):3123-3129.

22. Kovalevsky AY, Tie Y, Liu F, et al. Effectiveness of nonpeptide clinical inhibitor TMC-114 on HIV-1 protease with highly drug resistant mutations D30N, I50V, and L90M. J Med Chem. 2006;49(4): 1379-1387.

23. De Meyer S, Azijn H, Surleraux D, et al. TMC114, a novel human immunodeficiency virus type 1 protease inhibitor active against protease inhibitor-resistant viruses, including a broad range of clinical isolates. Antimicrob Agents Chemother. 2005;49(6):2314-2321.

24. Surleraux DL, Tahri A, Verschueren WG, et al. Discovery and selection of TMC114, a next generation HIV-1 protease inhibitor. J Med Chem. 2005;48(6):1813-1822.

25. King NM, Prabu-Jeyabalan M, Nalivaika EA, Wigerinck $P$, de Bethune MP, Schiffer CA. Structural and thermodynamic basis for the binding of TMC114, a next-generation human immunodeficiency virus type 1 protease inhibitor. J Virol. 2004;78(21):12012-12021.

26. McKeage K, Perry CM, Keam SJ. Darunavir: a review of its use in the management of HIV infection in adults. Drugs. 2009;69(4):477-503.

27. Koh Y, Matsumi S, Das D, et al. Potent inhibition of HIV-1 replication by novel non-peptidyl small molecule inhibitors of protease dimerization. J Biol Chem. 2007;282(39):28709-28720.

28. Dierynck I, De Wit M, Gustin E, et al. Binding kinetics of darunavir to human immunodeficiency virus type 1 protease explain the potent antiviral activity and high genetic barrier. J Virol. 2007;81(24):13845-13851. 
29. DeJesus E, Gottlieb MS, Gathe JC Jr, Greenberg ML, Guittari CJ, Zolopa AR. Safety and efficacy of enfuvirtide in combination with darunavir-ritonavir and an optimized background regimen in treatmentexperienced human immunodeficiency virus-infected patients: the below the level of quantification study. Antimicrob Agents Chemother. 2008;52(12):4315-9.

30. Agency EM. Prezista. URL: www.emea.europa.eu/humandocs/ PDFs/.../prezista/H-707-en1.pdf. 2009.

31. Back D, Sekar V, Hoetelmans RM. Darunavir: pharmacokinetics and drug interactions. Antivir Ther. 2008;13(1):1-13.

32. Sax PE. FDA approval: darunavir. AIDS Clin Care. 2006;18(8):71.

33. Rivero A, Camacho A, Perez-Camacho I, Torre-Cisneros J. [Darunavir in HIV/HVC/HVB coinfection]. Enferm Infecc Microbiol Clin. 2008; 26 Suppl 10:37-42.

34. Rachlis A, Clotet B, Baxter J, Murphy R, Lefebvre E. Safety, tolerability, and efficacy of darunavir (TMC114) with low-dose ritonavir in treatment-experienced, hepatitis B or C co-infected patients in POWER 1 and 3. HIV Clin Trials. 2007;8(4):213-220.

35. Yilmaz A, Izadkhashti A, Price RW, et al. Darunavir concentrations in cerebrospinal fluid and blood in HIV-1-infected individuals. AIDS Res Hum Retroviruses. 2009;25(4):457-461.

36. Clotet B, Bellos N, Molina JM, et al. Efficacy and safety of darunavirritonavir at week 48 in treatment-experienced patients with HIV-1 infection in POWER 1 and 2: a pooled subgroup analysis of data from two randomised trials. Lancet. 2007;369(9568):1169-1178.

37. Pozniak A, Opravil M, Beatty G, Hill A, de Bethune MP, Lefebvre E. Effect of baseline viral susceptibility on response to darunavir/ritonavir versus control protease inhibitors in treatment-experienced HIV type 1-infected patients: POWER 1 and 2. AIDS Res Hum Retroviruses. 2008;24(10):1275-1280.

38. Denes Banhegyi CK, Da Cunha C, Stefan Schneider S, et al. Phase III TITAN Week 96 final analysis: efficacy/safety of darunavir/ritonavir versus lopinavir/ritonavir in lopinavir/ritonavir-naïve, treatmentexperienced patients. 9th International Congress on Drug Therapy in HIV Infection; 2008; Glasgow, United Kingdom; 2008.

39. Smith CJ, Phillips AN, Dauer B, et al. Factors associated with viral rebound among highly treatment-experienced HIV-positive patients who have achieved viral suppression. HIV Med. 2009;10(1):19-27.

40. de Meyer S, Vangeneugden T, van Baelen B, et al. Resistance profile of darunavir: combined 24-week results from the POWER trials. AIDS Res Hum Retroviruses. 2008;24(3):379-388.

41. Madruga JV, Berger D, McMurchie M, et al. Efficacy and safety of darunavir-ritonavir compared with that of lopinavir-ritonavir at 48 weeks in treatment-experienced, HIV-infected patients in TITAN: a randomised controlled phase III trial. Lancet. 2007;370(9581): 49-58.
42. McKinnell JA, Lin HY, Nevin CN, et al. Early virologic suppression with three-class experienced patients: 24-week effectiveness in the darunavir outcomes study. AIDS. 2009. In press.

43. Ortiz R, Dejesus E, Khanlou H, et al. Efficacy and safety of once-daily darunavir/ritonavir versus lopinavir/ritonavir in treatment-naive HIV-1infected patients at week 48. AIDS. 2008;22(12):1389-13897.

44. Mills AM, Nelson M, Jayaweera D, et al. Once-daily darunavir/ ritonavir vs lopinavir/ritonavir in treatment-naive, HIV-1-infected patients: 96-week analysis. AIDS. 2009. In press.

45. Arribas JRAH, Gestoft J, Fatkenheuer G, et al; for the MONET study group. The MONET Trial: Darunavir/ritonavir monotherapy shows non-inferiorefficacy to standard HAART, for patients with HIV RNA $<50$ copies/ml at baseline. 5th IASConference; 2009; Cape Town, South Africa; 2009.

46. Katlama CVMA, Algarte-Genin et al. MONOI A randomised multicenter study to compare efficay of a monotherapy of darunavir to a triple therapy with 2 nucleoside analoguescombined to darunavir/r in HIV infected patients with full viral suppression. 5th IAS; 2009; Cape Town, South Africa; 2009.

47. Molina JM, Cohen C, Katlama C, et al. Safety and efficacy of darunavir (TMC114) with low-dose ritonavir in treatment-experienced patients: 24-week results of POWER 3. J Acquir Immune Defic Syndr. 2007;46(1):24-31.

48. Tomaka F, Lefebvre E, Sekar V, et al. Effects of ritonavir-boosted darunavir vs ritonavir-boosted atazanavir on lipid and glucose parameters in HIV-negative, healthy volunteers. HIV Med. 2009;10(5): 318-327.

49. McKinnell JA, Lin HY, Nevin CN, et al. Early virologic suppression with three-class experienced patients: 24-week effectiveness in the darunavir outcomes study. AIDS. 2009;23(12):1539-1546.

50. Hill A, Hemmett L, Wilson B. Analysis of costs by CD4 count category for the darunavir/r 600/100 mg bid and control protease inhibitor arms of the POWER 1 and 2 trials. HIV Clin Trials. 2007;8(5): 303-310.

51. Hill AM, Smith C. Analysis of treatment costs for HIV RNA reductions and CD4 increases for darunavir versus other antiretrovirals in treatment-experienced, HIV-infected patients. HIV Clin Trials. 2007;8(3):121-131

52. Chen RY, Accortt NA, Westfall AO, et al. Distribution of health care expenditures for HIV-infected patients. Clin Infect Dis. 2006;42(7):1003-1010.
HIV/AIDS - Research and Palliative Care

\section{Publish your work in this journal}

HIV/AIDS - Research and Palliative Care is an international, peerreviewed open-access journal focusing on advances in research in HIV, its clinical progression and management options including antiviral treatment, palliative care and public healthcare policies to control viral spread. The journal welcomes original research, basic science,

\section{Dovepress}

clinical \& epidemiological studies, reviews \& evaluations, expert opinion \& commentary, case reports \& extended reports. The manuscript management system is completely online and includes a very quick and fair peer-review system. Visit http://www.dovepress.com/ testimonials.php to read real quotes from published authors. 